\title{
Demenz durch Fernsehen
}

\section{Kognitive Beeinträchtigungen bei älteren Menschen durch übermäßigen TV-Konsum}

\author{
Korrespondenzadresse \\ Prof. Dr. Dr. Manfred Spitzer \\ Universität Ulm \\ Abteilung für Psychiatrie \\ Leimgrubenweg 12-14 \\ 87054 Ulm \\ Bibliografie \\ DOI https://doi.org/10.1055/a-0873-9009 \\ Nervenheilkunde 2019; 38: 363-371 \\ (c) Georg Thieme Verlag KG Stuttgart · New York \\ ISSN 0722-1541
}

\section{ZUSAMMENFASSUNG}

Das Fernsehen wurde als eine besondere kulturelle Aktivität beschrieben, bei der rasch wechselnde fragmentierte dichte sensorische Reize einerseits mit Passivität des Betrachters andererseits kombiniert sind. Aufgrund der genannten Charakteristika ist diese kulturelle Aktivität - wie viele andere kulturelle
Aktivitäten auch - möglicherweise gesundheitsschädlicher als bisher angenommen wurde. Dies betrifft nicht nur die Kindheit und Jugend, sondern auch und gerade das höhere Alter. Ab einer täglichen Nutzungsdauer von 3,5 Stunden hat nach dem gegenwärtigen Stand der Erkenntnis das Fernsehen bei Menschen im Alter von über 50 Jahren einen ungünstigen Einfluss auf den weiteren Verlauf der geistigen Leistungsfähigkeit. Der Zusammenhang ist nicht auf Bewegungsmangel oder die Verdrängung anderer gesunder Verhaltensweisen zurückzuführen und bleibt auch bei Berücksichtigung der üblichen demografischen und gesundheitlichen Variablen bestehen. Angesichts der gegenwärtigen demografischen Entwicklung wird ohnehin mit einer deutlichen Zunahme demenzieller Erkrankungen in den nächsten Jahrzehnten gerechnet, die unser Gesundheitssystem stark belasten wird. Der mit zunehmendem Alter deutlich zunehmende TV-Konsum verstärkt damit eine Entwicklung, der wir gegensteuern müssen. Es besteht daher dringender Forschungsbedarf im Hinblick auf unseren „alltäglichen“ und zugleich gesundheitsschädlichen Bildschirmmedienkonsum.
Über das Fernsehen macht sich heute kaum noch jemand Gedanken. Es ist einfach da, wie der Strom aus der Steckdose oder flieBendes Warm- oder Kaltwasser, und zählt zu den beliebtesten Freizeitbeschäftigungen der Deutschen. Laut der Arbeitsgemeinschaft Fernsehforschung (AGF) belief sich die durchschnittliche Fernsehdauer pro Tag im Jahr 2018 auf 217 Minuten, also 3,5 Stunden und 7 Minuten. In den letzten 10 Jahren schwankte dieser Wert zwischen 3,5 Stunden und 2 Minuten im Jahr 2009 und 3,5 Stunden und 15 Minuten im Jahr 2011 (dem „Allzeithoch“; [30]).

Nach wie vor gehört das Fernsehen also zu den am meisten benutzten Bildschirmmedien, wobei dies vor allem für die ältere Generation gilt. Wie $\mathbf{A b b} \mathbf{1}$ zeigt, nimmt der Fernsehkonsum der Deutschen mit dem Lebensalter zu: Während die 3- bis 13-Jährigen 45 Minuten pro Tag fernsehen, sind es bei den 14- bis 29-Jährigen schon 76 Minuten und bei allen über 14-Jährigen 216 Minuten, also etwas mehr als 3,5 Stunden.

Welche Konsequenzen hat Fernsehen für den Zuschauer? Interessanterweise gab es für Menschen im höheren Lebensalter bislang hierzu nur wenige Untersuchungen. Gute Daten lagen bislang vor allem für junge Menschen vor: Bei Kindern und Jugendlichen ist die Sache klar, wie durch über Jahrzehnte hinweg publizierte Studien aus vielen Ländern eindrücklich gezeigt werden konnte: Zuviel Fernsehen kann der kognitiven Entwicklung schaden und damit der Bildungsbiografie junger Menschen, wie schon früher in dieser Zeitschrift [20, 22] und von mir bereits im Jahr 2005 zusammenfassend in Buchform veröffentlicht worden war [21]. Dass der Fernsehkonsum in jungen Jahren damit einen Risikofaktor für die Entwicklung von Demenz im Alter darstellt, folgt zwangsläufig aus der Tatsache, dass eine geringere Bildung in jungen Jahren mithin einer der größten Risikofaktoren für die Entwicklung einer Demenz im Alter darstellt $[17,25]$.

Was den Fernsehkonsum im höheren Lebensalter anbelangt, war die Datenlage bislang längst nicht so klar. Man vermutete zwar bereits im Jahr 1993, dass die Kombination von „rasch wechselnden fragmentierten dichten sensorischen Reizen einerseits und Passivität des Betrachters andererseits “ zur Entwicklung einer Demenz beitragen könnte, hatte aber damals keine Belege dafür [1]. Dies änderte sich erst allmählich in den Jahren danach.

Bereits im Jahr 2005 publizierten US-amerikanische Wissenschaftler eine Fallkontrollstudie an 135 Patienten mit Demenz vom Alzheimer-Typ und 331 gesunden Kontrollpersonen [16]. Man verglich beide Gruppen im Hinblick auf 26 Freizeitaktivitäten, denen die Teilnehmer zwischen dem 40. und 59. Lebensjahrzehnt nachgegangen waren und fand mit jeder Stunde TV-Konsum täglich ein um Faktor 1,3 gesteigertes Risiko an einer Alzheimer Demenz erkrankt zu sein (unter Berücksichtigung der Kontrollvariablen Alter, Geschlecht, Einkommen und Bildung). Bereits damals führten die Autoren die Vermutung an, dass es die besonderen Eigenschaften des Fernsehens sind (geringe geistige Beanspruchung), die den Effekt verursachen könnten: „Unsere Befunde passen zu der Überlegung, dass die Teilnahme an nicht intellektuell stimulierenden Aktivitäten mit einem erhöhten Risiko, an Alzheimer Demenz zu er- 


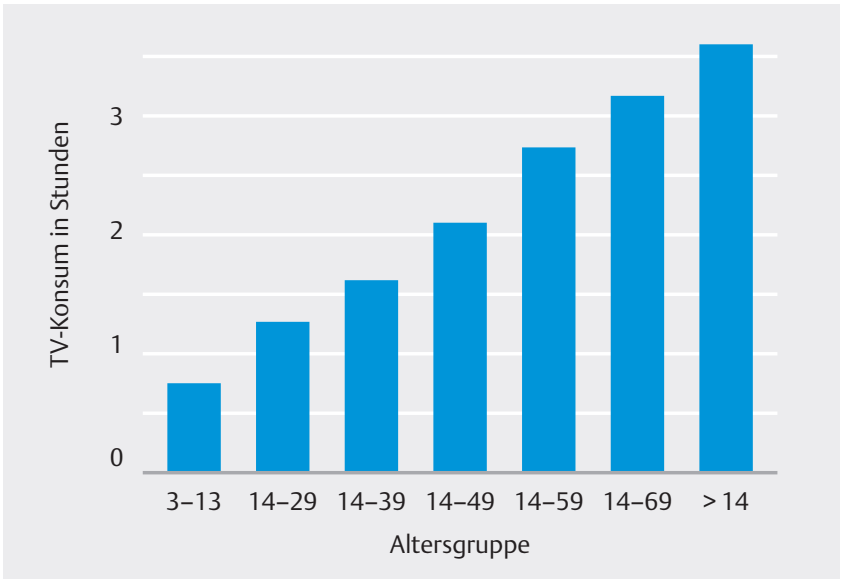

- Abb. 1 Je höher das Durchschnittsalter der Gruppe, desto länger wird ferngesehen. Man beachte, dass hier - abgesehen von der ersten Säule ganz links - nicht die TV-Zeiten in verschiedenen Altersgruppen für sich gezeigt sind, sondern jeweils die TV-Zeiten für die über 14-Jährigen bis zu einem bestimmten Alter, welches zunimmt. Trotz längerer Suche waren Zahlen für die einzelnen Gruppen jeweils für sich genommen für mich nicht auffindbar, bzw. nicht frei zugänglich [31].

kranken, verbunden ist, und dass Fernsehen einen Marker für die verminderte Teilnahme an intellektuell stimulierenden Aktivitäten darstellen könnte" 1 ([16], S. 157).

New Yorker Wissenschaftler gingen mit Unterstützung des National Institute of Aging in Bethesda, Maryland, der Frage nach, ob bestimmte TV-Programme einen besonderen negativen Einfluss auf die kognitive Leistungsfähigkeit haben [7]. Die befragten 289 Frauen im Alter von 70 -79 Jahren, die zu den gesünderen 2 Dritteln einer Gesamtkohorte detailliert nach ihren Fernsehgewohnheiten. Man fragte im Einzelnen ab, in welche Kategorien das gesehene TV-Programm fällt. Am häufigsten wurden Nachrichten geschaut; weitere Kategorien (in englischer Sprache²) waren „Detective, Talk, Soap, Game, Nature, Mysteries, Comedies, Sports, Movies, Other. “ Die Teilnehmerinnen waren im Mittel 75,5 Jahre alt und schauten 4 Stunden fern. Ihre kognitive Leistungsfähigkeit wurde dann mit bekannten neuropsychologischen Testverfahren gemessen (Trail Making Test, TMT; Hopkins Verbal Learning Test, Revised, HVLT-R; Mini-Mental State Examination, MMSE).

Insgesamt zeigte die Studie einen klaren Zusammenhang zwischen der Bevorzugung von Talkshows und Seifenopern („Talk“, „Soap“) mit dem Bestehen von Aufmerksamkeits- und Gedächtnisdefiziten. Um auszuschließen, dass es sich um einen Effekt von prodromal vorbestehenden depressiven oder demenziellen Erkrankungen handelt, wiederholte man daher die Analyse nur mit Daten von denjenigen Teilnehmerinnen mit intakter globaler kognitiver Leistungsfähigkeit (ausgeschlossen wurden $n=28$ ) und ohne jeg-

1 „Findings are consistent with the view that participation in non-intellectually stimulating activities is associated with increased risk of developing $A D$, and suggest television viewing may be a marker of reduced participation in intellectually stimulating activities."

2 In den USA wird das Fernsehen in etwas andere Kategorien eingeteilt als hierzulande, sodass ich die englischen Bezeichnungen der Kategorien übernommen habe. liche depressive Symptomatik (ausgeschlossen: $n=18$ ). Das Ergebnis blieb hierdurch unbeeinflusst, sodass die Autoren insgesamt zu dem Ergebnis kommen, dass eine Präferenz beim Fernsehen für Talkshows und Seifenopern mit einer etwa 7,3-fach bzw. 13,5-fach erhöhten Wahrscheinlichkeit von geringerer geistiger Leistungsfähigkeit einhergeht.

Da es sich um eine Querschnittsstudie handelt, lässt sich prinzipiell nicht sagen, wie dieser Zusammenhang zu interpretieren ist: Das Schauen von Talkshows und Seifenopern könnte zu Einbußen in der geistigen Leistungsfähigkeit führen; es könnte jedoch auch sein, dass Menschen mit eingeschränkter geistiger Leistungsfähigkeit diese TV-Programme bevorzugen, weil sie wenig kognitive Ansprüche stellen. Die Autoren geben weiterhin zu bedenken, dass diese Sendungen aufgrund ihres „parasozialen “3 Charakters zum Stressabbau geeignet seien und daher einen Marker für Stress darstellen könnten, was den Zusammenhang ursächlich erklären würde.

Eine im Fachblatt Neurology publizierte chinesische Studie erfasste 5437 Teilnehmer beiderlei Geschlechts im Alter von 55 Jahren oder mehr über einen Zeitraum von 5 Jahren (Chongqing Aging Study; [34]). Jährlich wurde mit der Mini-Mental State Examination (MMSE) die kognitive Leistungsfähigkeit bestimmt, wobei sich ein durchschnittliches Risiko von 2,3 \% für die Entwicklung einer kognitiven Leistungsbeeinträchtigung (cognitive impairment) zeigte. Gemessen wurden Lebensgewohnheiten bzw. geistige, körperliche und soziale Aktivitäten (Lesen, Brettspiele, Fernsehen, Radiohören, Schreiben, Gartenarbeit, Angeln, Verwandte und Freunde besuchen, Reisen, Malen und Zeichnen, Radiohören, Tanzen, Musizieren) sowie bekannte Risikofaktoren (Alkohol- und Tabakkonsum, das Bestehen körperlicher Erkrankungen oder einer Depression, geringer Bildungsgrad) für die Entwicklung kognitiver Beeinträchtigungen. Während Lesen und Brettspiele im Verlauf mit einer vergleichsweise geringeren kognitiven Beeinträchtigung signifikant einhergingen, zeigte sich der entgegengesetzte Zusammenhang für das Fernsehen.

Die Autoren diskutieren dieses Ergebnis vorsichtig erneut dahingehend, dass Fernsehen sowohl kognitive Beeinträchtigung zur Folge haben könnte oder aber auch als Zeichen für eine bereits bestehende Beeinträchtigung gewertet werden könne. Interessanterweise zeigten Musizieren, Schreiben sowie die verschiedenen körperlichen und sozialen Aktivitäten keinen Zusammenhang mit der Entwicklung kognitiver Defizite. Lediglich kognitiv anspruchsvolle Tätigkeiten (Lesen, Brettspiele) zeigten einen positiven Zusammenhang mit der geistigen Leistungsfähigkeit und Fernsehen einen negativen. Obwohl die Autoren aus ihren Daten keine Kausalität ableiten können, halten sie den Zusammenhang dennoch für klinisch relevant: Sofern man während der Anamnese ohnehin über Freizeitund Mediennutzungsverhalten spricht, sollte man etwas genauer nach den geschauten Sendungen fragen. Werden dann vor allem Talkshows und Seifenopern angegeben, könne dies im Einzelfall eine genauere Betrachtung der geistigen Leistungsfähigkeit nahelegen.

3 Die Autoren verweisen in diesem Zusammenhang auf eine ganze „Theorie“ parasozialer Interaktionen, die durch große Nähe zu und große Aufmerksamkeit für fremde Personen gekennzeichnet ist, die man häufig in Medien sieht. Ich gehe dem hier nicht weiter nach, möchte es jedoch nicht unerwähnt lassen, weil im Folgenden ähnliche Gedanken nochmals Erwähnung finden. 
Eine aufwendige, im Fachblatt JAMA Psychiatry publizierte Längsschnittstudie ergab weitere Hinweise für ungünstige Auswirkungen von geringer körperlicher Aktivität und viel Fernsehen in jüngeren Jahren (18- bis 30-Jährige, Durchschnittsalter 25, 1 Jahre; $56,5 \%$ weiblich; $54,5 \%$ hellhäutig) auf die geistige Leistungsfähigkeit 25 Jahre später [10]. Die Studie wurde in 4 US-amerikanischen Großstädten in den Jahren 1985-2011 durchgeführt und beinhaltet Daten (alle 5 Jahre wurde gemessen) zu 3247 Teilnehmern (man hatte mit 5115 begonnen). Zur Messung der körperlichen Aktivität wurde ein standardisierter Fragebogen verwendet, der 13 Aktivitäten starker und moderater körperlicher Anstrengung einzeln erfasst. Der tägliche TV-Konsum wurde erfragt, und die geistige Leistungsfähigkeit nach 25 Jahren wurde mittels dreier standardisierter Verfahren gemessen: Der Digital Symbol Substitution Test (DSST) diente zur Erfassung von Schnelligkeit und exekutiven Funktionen, für die zudem auch der Stroop-Test zum Einsatz kam. Das verbale Gedächtnis wurde mit dem Rey Auditory Verbal Learning Test (RAVLT) gemessen. Eine ganze Reihe von möglicherweise relevanten weiteren Kovariablen (Alter, Geschlecht, ethnische Zugehörigkeit, Bildungsgrad, BMI, Alkohol- und Nikotinkonsum und Bluthochdruck) wurden ebenfalls erfasst.

Gruppenvergleiche im Hinblick auf die körperliche Aktivität ergaben für die exekutiven Funktionen ein um etwa $50 \%$ gesteigertes Risiko (DSST: OR 1,62; Stroop: OR: 1,37) der Abnahme in der Gruppe mit geringer körperlicher Aktivität ( $n=528$, entsprechend 16,7 \% der Gesamtgruppe; alle Ergebnisse signifikant). Das verbale Gedächtnis war nicht beeinträchtigt (RAVLT: OR 1,17, n. s.). Bezog man die genannten Kovariablen mit in die Analyse ein, blieben die Ergebnisse für den DSST (OR 1,47) signifikant.

Die Gruppe mit viel TV-Konsum ( $n=353$, entsprechend 10,9\% der Gesamtgruppe; nur signifikante Ergebnisse werden berichtet) hatte dagegen ein um 50 bis mehr als $100 \%$ gesteigertes Risiko einer Reduktion der exekutiven Funktionen bei allen 3 Tests (DSST: OR 2,26; Stroop. OR: 2,01; RAVLT: OR 1,58). Unter Einbeziehung der Kovariablen reduzierten sich diese Werte etwas, blieben jedoch für den DSST (OR 1,64) und den Stroop-Test (OR 1,58) signifikant. Auch unter Einbeziehung der körperlichen Aktivität in das Regressionsmodell änderten sich diese Ergebnisse nur unerheblich. Weiterhin gab es keine signifikante Interaktion zwischen körperlicher Aktivität und TV-Konsum.

In dieser Studie wurde deutlich, dass der negative Effekt des TV-Konsums mit der Verdrängung körperlicher Aktivität allein nicht erklärt werden kann. Zudem erwies sich der Effekt des Fernsehens als größer als der Effekt geringer körperlicher Aktivität und als weitgehend unabhängig von diesem. Ein wesentlicher Nachteil der Studie besteht darin, dass keine Ausgangsmessung der geistigen Leistungsfähigkeit vorgenommen wurde und man daher nicht ausschließen kann, dass die geistig Schwächeren mehr Fernsehen und damit der Ursache-Wirkungs-Zusammenhang nicht vom Fernsehen zur geringen geistigen Leistungsfähigkeit geht, sondern umgekehrt verläuft ${ }^{4}$. Weil jedoch die Teilnehmer dieser Studie zu Beginn im Mittel 25 Jahre alt waren, ist es unwahrscheinlich, dass man hier schon Einschränkungen (im Sinne von Anzeichen einer Demenz) hätte messen können. Dennoch ist eine solche umgekehrte Kausalität im Hinblick auf mehr TV-Konsum und geringere geistige

4 Im Englischen spricht man von „reverse causality“.
Leistungsfähigkeit (das zweite bewirkt das erste) allerdings nicht mit letzter Sicherheit auszuschließen, denn man weiß aus anderen Studien, dass weniger gebildete Menschen mehr Fernsehen, und dass die Bildung eines Menschen vor Demenz schützt (siehe hierzu auch die Diskussion).

Eine sehr große britische Studie (UK Biobank cohort; [2]) fand ebenfalls einen negativen Zusammenhang zwischen TV-Konsum und geistiger Leistungsfähigkeit 5 Jahre später. Die großen Teilnehmerzahlen (je nach Test zwischen gut 12000 und gut 114000) werden allerdings durch eine weniger genaue Methodik (Selbstbeurteilung und Tests am Computer zuhause, d. h. unbeobachtet) gleichsam „erkauft“, was eigene Probleme bei der Interpretation der Daten mit sich bringt.

Ganz aktuell wurde jetzt eine Studie über die Auswirkungen des TV-Konsums auf die geistige Leistungsfähigkeit im Alter veröffentlicht, die wegen des aufwendigen methodischen Vorgehens bei zugleich recht großer Stichprobe als bemerkenswert einzustufen ist [6]. Man verwendete Daten von 3590 Teilnehmern im Alter von über 50 Jahren, die geistesgesund (also ohne Demenz) in den Jahren 2008 und 2009 in die English Longitudinal Study of Aging (ELSA) aufgenommen worden waren und 6 Jahre danach (20142015) erneut untersucht wurden.

Die Teilnehmer waren im Mittel 67 Jahre alt (Bereich: 52 bis über 90 Jahre), zu 43,7\% männlich und zu 72,3\% verheiratet bzw. fest liiert. Die meisten arbeiteten nicht mehr, aber immerhin etwa jeweils $15 \%$ arbeiten noch vollzeitig bzw. in Teilzeit. Nach ihrem durchschnittlichen täglichen Fernsehkonsum wurden die Teilnehmer in 5 etwa gleich große Gruppen (Quintile) eingeteilt, die

- weniger als 2,5 Stunden (19,6\%),

- 2,5-3,5 Stunden (19,1\%),

- 3,5-4,5 Stunden (18,4\%),

- 4,5-7 Stunden (23,4\%) bzw.

- mehr als 7 Stunden (19,6\%) täglich fern sahen.

Wie $>$ Tab. 1 zeigt, schauen Frauen bzw. allein lebende, nicht mehr arbeitende, weniger gebildete und ärmere Menschen mehr fern als Männer bzw. verheiratete, arbeitende, gebildetere und wohlhabendere Menschen ( $\mathbf{A} \mathbf{b} \mathbf{b} . \mathbf{2}$ ).

Zur Messung der geistigen Leistungsfähigkeit der Teilnehmer wurden bekannte Textverfahren für das Neulernen verbaler Information (verbal memory) und die Wortflüssigkeit (semantic fluency) zu Beginn der Studie (baseline) und 6 Jahre später verwendet. Zur Messung des verbalen Gedächtnisses wurden 10 Wörter per Tonaufzeichnung (ein Wort jede zweite Sekunde) dargeboten und sowohl gleich danach sowie einige Zeit später wieder abgefragt. Die Leistung aus beiden Abfragen diente als Maß des verbalen Gedächtnisses bzw. des Neulernens. Die semantische Flüssigkeit wurde gemessen, indem die Teilnehmer so viele Tiere wie möglich in einer Minute nennen sollten.

Dann wurde der Einfluss der Zeit des Fernsehkonsums mit 3 Regressionsmodellen untersucht. Man verglich hierzu, um wie viel sich die Leistungsfähigkeit in den Tests bei den Teilnehmern aus den Quintilen 2-5 gegenüber dem ersten Quintil (weniger als 2,5 Stunden TV-Konsum) verändert hat. Dieses Vorgehen entspricht dem von nahezu allen Studien zu den Auswirkungen des Fernsehkonsums auf was auch immer - Bildung, Körpergewicht, Kriminalität etc. Es ist der Tatsache geschuldet, dass es praktisch niemanden 
- Tab. 1 Deskriptive Ergebnisse in den 5 Quintilen nach zunehmender Fernsehzeit im Hinblick auf demografische und andere untersuchte Variablen (nach [6], Table 1). Sämtliche Unterschiede in den hier angeführten Variablen (Geschlecht, Status etc.) bezüglich der Quintile sind mit p<0,001 signifikant.

\begin{tabular}{|c|c|c|c|c|c|c|}
\hline \multirow[t]{2}{*}{ Variable } & & \multicolumn{5}{|c|}{ Quintile nach täglicher TV-Zeit } \\
\hline & & $<2,5 h$ & $2,5-3,5 h$ & $3,5-4,5 h$ & $4,5-7 \mathrm{~h}$ & $>7 \mathrm{~h}$ \\
\hline \multirow[t]{2}{*}{ Geschlecht } & $\mathrm{m}$ & $23,8 \%$ & $20,5 \%$ & $16,8 \%$ & $20,8 \%$ & $18 \%$ \\
\hline & w & $16,2 \%$ & $18 \%$ & $19,6 \%$ & $25,5 \%$ & $20,7 \%$ \\
\hline \multirow[t]{2}{*}{ Status } & verh/gem & $21,1 \%$ & $19,8 \%$ & $18,1 \%$ & $22,3 \%$ & $18,8 \%$ \\
\hline & allein & $15,6 \%$ & $17,4 \%$ & $19,0 \%$ & $26,5 \%$ & $21,5 \%$ \\
\hline \multirow[t]{3}{*}{ Arbeit } & Vollzeit & $28,2 \%$ & $23,6 \%$ & $16,1 \%$ & $16,5 \%$ & $15,7 \%$ \\
\hline & Teilzeit & $24,2 \%$ & $20,1 \%$ & $19,9 \%$ & $17,7 \%$ & $18,1 \%$ \\
\hline & keine & $16,7 \%$ & $18,0 \%$ & $18,5 \%$ & $26.2 \%$ & $20,7 \%$ \\
\hline \multirow[t]{4}{*}{ Bildung } & kein Abschluss & $7,6 \%$ & $12,7 \%$ & $16,8 \%$ & $32,5 \%$ & $30,4 \%$ \\
\hline & Abschluss mit 16 & $16,9 \%$ & $19,6 \%$ & $21,0 \%$ & $23,6 \%$ & $19,0 \%$ \\
\hline & Abschluss mit 18 & $19,1 \%$ & $21,5 \%$ & $19,3 \%$ & $22,8 \%$ & $17,3 \%$ \\
\hline & höherer Abschluss & $32,3 \%$ & $25,9 \%$ & $16,6 \%$ & $15,3 \%$ & $9,9 \%$ \\
\hline \multirow[t]{2}{*}{ SES } & $20 \%$ arm & $8,2 \%$ & $11,3 \%$ & $17,1 \%$ & $32,9 \%$ & $30,4 \%$ \\
\hline & $20 \%$ reich & $32.2 \%$ & $25,9 \%$ & $16,6 \%$ & $15,3 \%$ & $9,9 \%$ \\
\hline \multirow{3}{*}{$\begin{array}{l}\text { körperliche } \\
\text { Aktivität }\end{array}$} & Gering & $12,5 \%$ & $15,1 \%$ & $17,5 \%$ & $29,8 \%$ & $25,2 \%$ \\
\hline & Mäßig & $19,1 \%$ & $20,0 \%$ & $19,0 \%$ & $23,4 \%$ & $18,8 \%$ \\
\hline & Hoch & $28,2 \%$ & $21,4 \%$ & $17,8 \%$ & $16,5 \%$ & $16,2 \%$ \\
\hline
\end{tabular}
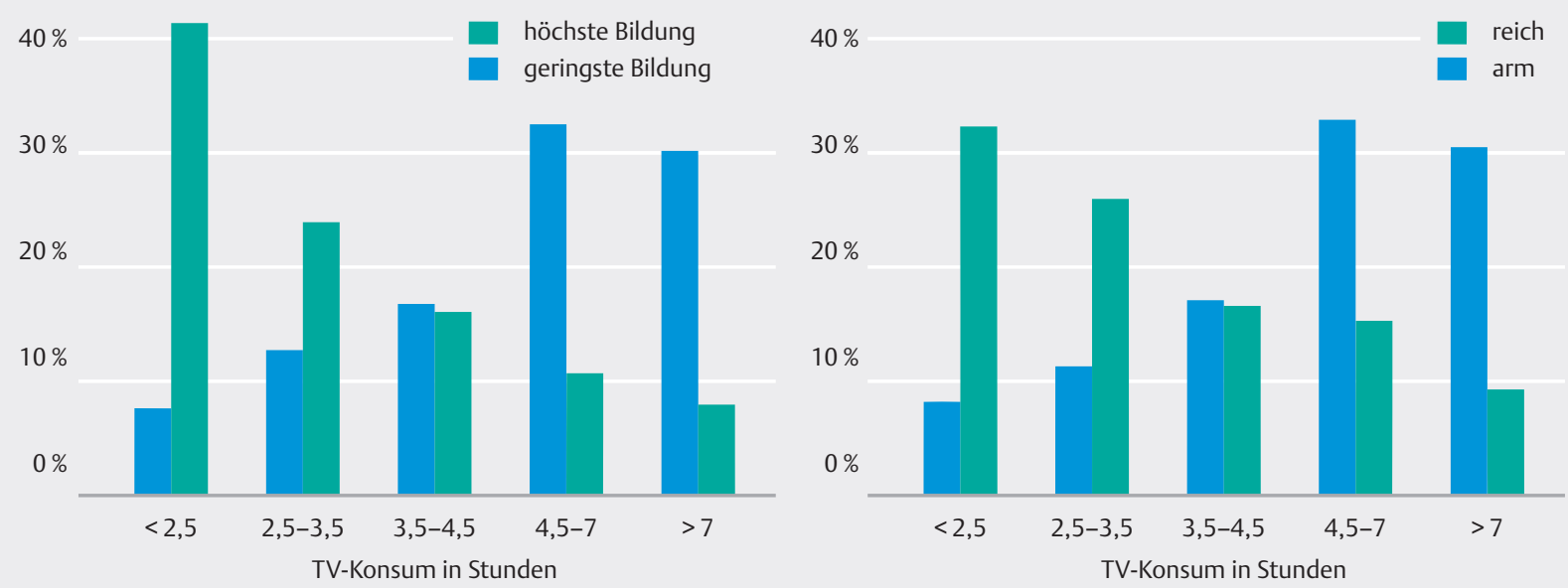

- Abb. 2 Daten aus $>$ Tab. 1 zum Zusammenhang von TV-Konsum in Stunden pro Tag und Bildung (kein Abschluss versus akademischer Abschluss) sowie sozioökonomischem Status (arm versus reich), wobei jeweils die Extremgruppen (oberstes und unterstes Fünftel) miteinander verglichen werden. Je gebildeter ein Mensch ist, desto weniger sieht er fern. Je ungebildeter er ist, desto mehr sieht er fern. Fast das gleiche Ergebnis (die Zahlen in der Tabelle sind teilweise fast identisch) liefert die Einteilung nach sozioökonomischem Status (nach Daten aus \ Tab. 1).

gibt, der überhaupt nicht fernsieht. Zum Vergleich: Will man die Auswirkungen des Rauchens auf die Gesundheit der Menschen untersuchen, dann kann man Raucher mit Nichtrauchern vergleichen. Dies gelingt bei Studien zum TV-Konsum nicht, weil praktisch nie- mand gar nicht fernsieht. Man ist also immer auf den Vergleich von mehr mit weniger Fernsehen angewiesen ${ }^{5}$.

5 Dies hat u. a. zur Konsequenz, dass man bis heute für den TV-Konsum keinen Schwellenwert angeben kann, ab dem er gefährlich werden könnte, weil die meisten Studien nicht im Längsschnitt durchgeführt worden waren und daher auch keinen Vergleich mit „baseline“ zuließen [21, 22]. In der vorliegenden Studie ist dies anders (siehe weiter unten im Haupttext). 


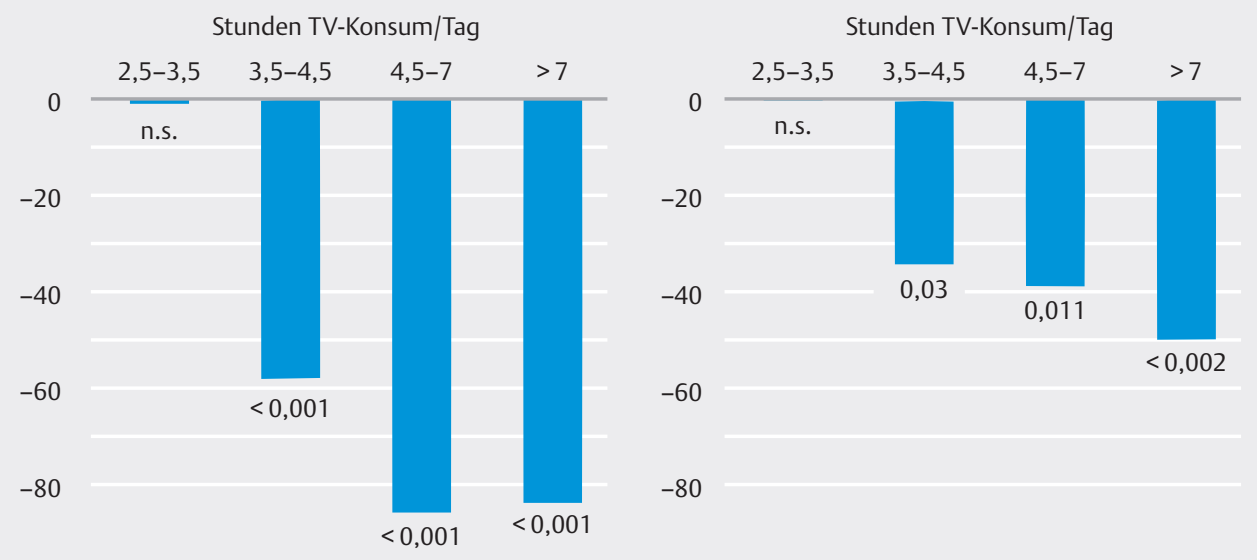

- Abb. 3 Abnahme des verbalen Gedächtnisses in Abhängigkeit vom Fernsehkonsum: Dargestellt ist die Abnahme des verbalen Gedächtnisses bei den 4 Gruppen mit längeren täglichen TV-Zeiten bezogen auf die Gruppe mit der geringsten TV-Nutzungszeit von 0-2,5 Stunden täglich; angegeben sind die beta-Gewichte der ersten beiden Regressionsmodelle (links: Modell 1; rechts: Modell 2). Die Abnahme in der Gruppe mit 2,5-3,5 Stunden Nutzungszeit ist in beiden Modellen nicht statistisch signifikant (n. s.). In den 3 Gruppen mit noch höherem TV-Konsum jedoch erreicht die Abnahme der verbalen Gedächtnisleistung in beiden Modellen jeweils das angegebene Signifikanzniveau ( $p$-Werte in der Abbildung angegeben; nach Daten aus [6], Table 2, linke Spalte). Zwischen den Modellen 2 und 3 gab es nur sehr geringe Unterschiede, weswegen die Daten aus Modell 3 nicht abgebildet sind.

Die Regressionsmodelle bezogen inkrementell zusätzliche Variablen mit ein: Mit Modell 1 untersuchte man die Auswirkungen des TV-Konsums auf die Messungen der geistigen Leistungsfähigkeit nach 6 Jahren unter Einbeziehung der Ausgangswerte (baseline). Bei Modell 2 bezog man noch zusätzlich die Auswirkungen demografischer Variablen (Geschlecht, Alter, Status, ethnische Zugehörigkeit, Arbeit, Bildung und sozioökonomischer Status, SES) mit ein sowie zusätzlich erfasste Variablen zur Gesundheit der Teilnehmer (Bestehen einer Depression, Selbstbeurteilung der körperlichen Gesundheit, Alkohol- und Nikotinkonsum, Vorliegen chronischer Erkrankungen, einschließlich kardiovaskulärer Probleme). In einem dritten Ansatz (Modell 3) wurde schließlich noch zusätzlich die körperliche Aktivität berücksichtigt.

Die 3 Modelle berücksichtigen also entweder

- nur den Zusammenhang zwischen Fernsehkonsum und Veränderung der geistigen Leistungsfähigkeit im Vergleich zur Ausgangsmessung, oder sie bezogen noch

- demografische und Gesundheitsdaten in die Auswertung mit ein bzw.

- noch zusätzlich das Ausmaß der körperlichen Aktivität.

Beim verbalen Gedächtnis zeigte sich in Modell 1 ein klarer dosisabhängiger Zusammenhang zum TV-Konsum: Je mehr Stunden täglich TV konsumiert wird, desto mehr nahm das verbale Gedächtnis im Vergleich zum Ausgangswert 6 Jahre zuvor ab ( $\triangleright$ Abb. $\mathbf{3}$ ). In den anderen beiden Modellen erwies sich dieser Zusammenhang zwar als 44-55\% kleiner, war aber dennoch deutlich: Selbst in Modell 3 ergab sich ein linearer (also dosisabhängiger) negativer Zusammenhang $(B=-0,13, S E=0,04, p<0,001, C l:-0,20$, $-0,06)$ zwischen täglicher Fernsehzeit und verbalem Gedächtnis. Zum Vergleich führen die Autoren an, dass nach ihren Berechnungen 3,5 Stunden TV-Konsum für das verbale Gedächtnis schädlicher sind als Armut (unteres Quintil verglichen mit dem mittleren
Quintil). Mehr als 7 Stunden täglicher TV-Konsum ist vergleichsweise schädlicher als nicht vorhandener Schulabschluss.

Im Hinblick auf die Wortflüssigkeit ergab das Model 1 ebenfalls eine negative Beziehung zu mehr als 3,5 Stunden täglichem TV-Konsum, der sich jedoch bei den Modellen 2 und 3 stark abschwächte und nicht mehr überzufällig war. Zwischen den beiden Modellen gab es nur kleine, numerische Unterschiede, sodass die Autoren davon ausgehen, dass vor allem die demografischen und die Gesundheitsvariablen für die Abschwächung verantwortlich sind, nicht jedoch das Ausmaß körperlicher Aktivität.

Weitere statistische Analysen zeigten, dass der Effekt nicht durch einen anderen Mechanismus (etwa einen bestimmten Risikofaktor oder eine bestimmte Krankheit) zustande kam. Es zeigte sich ferner, dass die vor dem Fernseher verbrachte Zeit bei den Teilnehmern über die Zeit hinweg recht stabil war, und dass, wenn der Effekt für den schmalen Bereich von 3-3,5 Stunden berechnet wurde, er sich noch nicht zeigt. Hieraus schließen die Autoren, dass 3,5 Stunden täglicher TV-Konsum als ein Schwellenwert zu betrachten ist, oberhalb dessen sich negative Auswirkungen von TV-Konsum bemerkbar macht.

Die Studie zeigt zunächst einmal, dass man die Effekte des Fernsehens nicht einfach als Verdrängung gesünderer Verhaltensweisen als Sitzen betrachten kann. Diese sogenannte Verdrängungshypothese [19] ist die schwächste Interpretation der Effekte von Bildschirmmedien, weil sie unmittelbar plausibel ist (wer vor dem Fernseher sitzt, tut vieles andere Gute bzw. Gesunde nicht) und ohne jegliche Vorannahmen zu gesundheitlichen Problemen durch Bildschirmmedien auskommt. (Motto: Bildschirmmedien sind an sich nicht schädlich, aber wenn man zu viel Zeit damit verbringt, macht man manches, was einem gut täte, weniger.) Gemäß dieser Hypothese könnte man vermuten (und so wurde es auch immer wieder behauptet), dass das Fernsehen (oder andere Bildschirmmedien wie Computer oder Spielekonsolen, Tabletts oder Smartphones) nicht per se schädliche Auswirkungen hätten, sondern le- 
diglich Bewegungsarmut begünstigten. Deswegen käme es dann zu den negativen Auswirkungen.

Dieser Mechanismus existiert einerseits sicherlich in der realen Welt, er erklärt jedoch die vorliegenden Daten nicht. Denn es wurde ja - und das ist eine Besonderheit der Studie - ein Zusammenhang zwischen TV-Konsum und Verminderung der geistigen Leistungsfähigkeit gefunden, der sich als unabhängig vom Ausmaß der körperlichen Bewegung darstellte. Zuviel Fernsehen scheint also über Bewegungsmangel mit all seinen Folgen hinausgehend zu einer Beeinträchtigung der geistigen Leistungsfähigkeit zu führen. Wer also meint, er brauche zwischen den Zeiten seines TV-Konsums nur gelegentlich zu joggen und wäre damit automatisch vor negativen Folgen geschützt, der irrt.

Wenn die Ursachen für den Zusammenhang zwischen Fernsehen und geistiger Leistungsfähigkeit nicht im Bewegungsmangel liegen, worin bestehen sie dann? Die Autoren diskutieren 3 mögliche Mechanismen:

- der passive Charakter des TV-Konsums,

- Stress (durch Fernsehen) mit konsekutivem neuronalen Zelltod im Hippocampus sowie

- die Verdrängung anderer Freizeitaktivitäten wie gemeinsames Spielen oder andere kulturelle bzw. soziale Aktivitäten, die der Entwicklung einer Demenz entgegenwirken ${ }^{6}$.

\section{Passiver Charakter des TV-Konsums}

Fernsehen ist ein passives Medium, das - sicherlich je nach Sendungsformat - nur wenig bis gar keine höheren kognitiven Tätigkeiten des Zuschauers verlangt. Selbst Dokumentationen sind inhaltlich so aufbereitet, dass sie leicht verständlich sind und man dem linearen Ablauf der Informationen schon beinahe „nebenher“ folgen kann. Im Unterschied zum Lesen beispielsweise sind die Bilder bereits da, visuelles und auditives System werden bedient wie ein Konsument im Supermarkt, wo die angebotenen Waren den Speiseplan vorgeben.

\section{Stress}

Wer sich vor dem Fernseher ausruhen möchte, hatte möglicherweise zuvor zu viel Stress erlebt. Fragt man die Leute, so ist „Stressabbau“ eines der am häufigsten angegebenen Motive zur Nutzung von Fernsehen und auch anderer Bildschirmmedien in der freien Zeit. Aber funktioniert das auch? Man darf durchaus bezweifeln, dass die weithin das TV-Programm füllenden Darstellungen von Gewalt und Horror tatsächlich die Konzentrationen von Noradrenalin oder Kortisol im Blut senken. Die Studienlage hierzu erscheint mir sehr dürftig, was angesichts der Relevanz (und der von der Allgemeinheit zu tragenden Kosten!) für einen dringenden Forschungsbedarf spricht. Neben dem hier dargelegten direkten Mechanismus mit TV-Konsum als Stressor, wäre allerdings auch zu fragen, ob die Menge täglichen TV-Konsums mit dem Ausmaß des Alltagsstress

6 Aus den Studien zu den Auswirkungen des Fernsehens bei Mäusen [3, 4] könnte man noch Unaufmerksamkeit, Hyperaktivität, geringere Neugierde geringeres Lernvermögen und erhöhte Risikobereitschaft erwähnen, auch diese Daten beziehen sich jedoch auf Mäuse in Entwicklung.

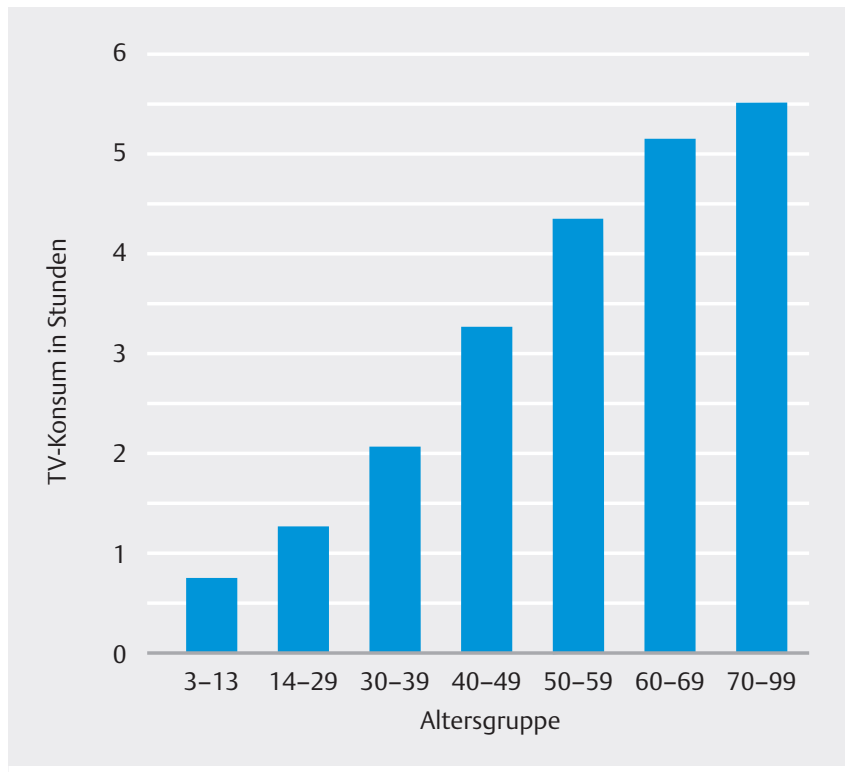

- Abb. 4 Täglicher Fernsehkonsum in Deutschland in Abhängigkeit vom Lebensalter (berechnet aus Daten nach [31]. Je höher das Lebensalter, desto länger wird ferngesehen.

im Zusammenhang steht. Wer tagsüber viel Stress erlebt, versucht am Abend durch längeres Fernsehen sich hiervon zu erholen. Insofern wäre dann die Dauer von TV-Konsum ein Parameter, der über erlebten Alltagsstress informiert, wenn diese „Erholungs- oder Kompensations-Hypothese" zuträfe.

\section{Verdrängung anderer Freizeitaktivitäten}

Es ist eine Binsenweisheit, dass der Fernsehkonsum andere Aktivitäten verdrängt. Hierbei handelt es sich keineswegs nur um Bewegung, sondern vor allem auch um gemeinsame Freizeitaktivitäten und damit um Sozialkontakte, die in unserer von Bildschirmmediennutzung geprägten Welt immer seltener werden: Bei Erwachsenen in den USA beträgt die Bildschirmmediennutzung im täglichen Durchschnitt 9 Stunden und 22 Minuten, davon eine Stunde und 39 Minuten bei der Arbeit und 7 Stunden und 43 Minuten in der Freizeit. 51 \% der Erwachsenen geben an, mehr als 8 Stunden täglich mit Medien zu verbringen, nur 19\% geben dagegen weniger als 4 Stunden tägliche Medienzeit an [13]. Da bleibt für soziales Erleben und die damit verbundenen kognitiven Herausforderungen kaum noch Zeit. Auch kann hinzutreten, dass die Verringerung der Häufigkeit und Dauer sozialer Kontakte zu einer Zunahme des Erlebens von Einsamkeit führen, von der wir heute wissen, dass Einsamkeit wiederum als Stressor bedeutsame negative Auswirkungen auf Gesundheit und Wohlbefinden haben kann ([12, 11]; zusammenfassende Darstellung in [28, 29] ).

Zurück zum Befund, dass Fernsehen im Alter mit verminderter geistiger Leistungsfähigkeit ab 3,5 Stunden pro Tag (im Vergleich zu bis zu 2,5 Stunden) im Zusammenhang steht. Ein flüchtiger Blick auf \ Abb. 1 könnte im Lichte dieses Ergebnisses zunächst als „Entwarnung“ interpretiert werden, scheinen wir doch im Wesentlichen weniger als 3,5 Stunden täglich fern zu sehen. Man kann jedoch aus der Abbildung bereits erahnen, dass ältere Menschen einen höheren TV-Konsum haben, denn je mehr von ihnen hinzu- 
kommen und je älter diese sind, desto stärker erhöht sich der Gesamtdurchschnitt.

Berechnet man die Daten aus $\mathbf{A b b} \mathbf{1}$ unter Zuhilfenahme der demografischen Daten zu Deutschland (wie viele Menschen sind gerade wie alt) neu, so lassen sich Altersgruppen mit klaren Kategoriengrenzen berechnen und es ergeben sich die in $\mathbf{A b b} \mathbf{4}$ dargestellten Werte. Wie man sieht, sind genau diejenigen, die in der Studie von Fancourt und Steptoe (2019) [6] untersucht worden waren, auch am stärksten betroffen: Der durchschnittliche Fernsehkonsum liegt in genau den Altersgruppen über 50 Jahren deutlich über 3,5 Stunden, für die in dieser Studie auch ein negativer Zusammenhang des täglichen TV-Konsums von über 3,5 Stunden mit geistiger Leistungsfähigkeit gefunden worden war. Bei den 50 bis 59-Jährigen liegt der tägliche TV-Konsum bei knapp 4,5 Stunden, bei den 60- bis 69-Jährigen bei gut 5 Stunden und bei den über 70-Jährigen bei gut 5,5 Stunden. So betrachtet sind die Ergebnisse praktisch sehr bedeutsam.

Aber ist der aus dem vereinigten Königreich stammende Befund auch für Deutschland relevant? Ohne auf die qualitativen Unterschiede des Fernsehens zwischen uns und unseren britischen Nachbarn eingehen zu können (das Parlament ist dort auf jeden Fall kurzweiliger, das haben wir alle in den letzten Wochen und Monaten gelernt), liegen Deutschland und Großbritannien im europäischen Vergleich - also quantitativ - nicht sehr weit auseinander, wie aus $>$ Tab. 2 hervorgeht. Die Deutschen schauen eher noch etwas mehr fern als die Briten, sodass die Ergebnisse aus Großbritannien durchaus auf uns angewandt werden können.

Der tägliche Fernsehkonsum in Europa schwankt zwischen 2 Stunden in der deutschen Schweiz und 5,25 Stunden in Rumänien (EU-Durchschnitt: 3 Stunden 47 Minuten). Wer nun denkt: „Da sind wir ja in Deutschland mit unserem durchschnittlichen täglichen TV-Konsum von 3 Stunden und 41 Minuten gerade nochmal halbwegs davon gekommen; sollen doch erst einmal die Rumänen, Serben, Portugiesen, Griechen, Türken, Polen oder Italiener nicht so viel fernsehen", der liegt falsch. Denn ein durchschnittlicher TV-Konsum von 3 Stunden 41 Minuten sagt auch, dass etwa die Hälfte der Menschen mehr als 3,5 Stunden vor dem Fernseher verbringt ${ }^{7}$. Und zweitens liegen die älteren Menschen, also genau die Gruppe, die bei erhöhtem TV-Konsum mit dosisabhängig häufiger an Demenz erkrankt, weit oberhalb der 3,5 Stunden.

Muss man sich um Länder mit durchschnittlich weit über 3,5 Stunden liegendem TV-Konsum Sorgen machen? - Vielleicht. Wie ein flüchtiger Blick auf die $>$ Tab. 2 vermuten lässt, gibt es eine deutliche negative Korrelation zwischen dem durchschnittlichen TV-Konsum der Bewohner eines Landes und deren wirtschaftlicher Leistungsfähigkeit (Bruttoinlandsprodukt pro Kopf). Berechnet man diese Korrelation, dann liegt sie kaufkraftbereinigt bei $r=-0,74\left(p<10^{-5}\right)$, nicht kaufkraftbereinigt liegt sie mit $r=-0,79$ sogar noch etwas höher. Nun ist Korrelation nicht gleich Kausalität.

7 Dies ist genau genommen nur dann der Fall, wenn man nicht den Mittelwert, sondern den Median zur Spaltung der Gruppe zugrunde legt. Daher schreibe ich „etwa“. 
- Tab. 2 TV-Konsum in Minuten pro Tag nach Ländern im Jahr 2017 [32]. Die Tabelle wurde um die wirtschaftliche Leistungsfähigkeit des betreffenden Landes (Bruttoinlandsprodukt pro Einwohner, unbereinigt sowie kaufkraftbereinigt) ergänzt (Daten aus Wikipedia zum Jahr 2017).

\begin{tabular}{|c|c|c|c|}
\hline Land & $\begin{array}{l}\text { TV Minuten/ } \\
\text { Tag }\end{array}$ & BIP/Kopf & $\begin{array}{l}\text { BIP/Kopf } \\
\text { (kaufkraft- } \\
\text { bereinigt) }\end{array}$ \\
\hline Rumänien & 317 & 10,757 & 24,508 \\
\hline Serbien & 313 & 5,899 & 15,000 \\
\hline Portugal & 284 & 21,161 & 30,417 \\
\hline Ungarn & 282 & 15,531 & 29,474 \\
\hline Nordmazedonien & 274 & 5,474 & 14,914 \\
\hline Griechenland & 262 & 18,637 & 27,737 \\
\hline Türkei & 261 & 10,512 & 26,893 \\
\hline Kroatien & 260 & 13,138 & 24,423 \\
\hline Polen & 259 & 13,822 & 29,523 \\
\hline Bulgarien & 249 & 8,064 & 21,687 \\
\hline Italien & 244 & 31,984 & 38,140 \\
\hline Estland & 243 & 19,840 & 31,749 \\
\hline Belgien (Süd) & 243 & 43,582 & 46,553 \\
\hline Russland & 242 & 10,608 & 27,835 \\
\hline Ukraine & 242 & 2,583 & 8,713 \\
\hline Slowakei & 237 & 17,664 & 33,025 \\
\hline Litauen & 236 & 16,730 & 32,229 \\
\hline EU & 227 & 32,700 & 32,700 \\
\hline Spanien & 225 & 28,359 & 38,286 \\
\hline Weißrussland & 225 & 5,760 & 18,931 \\
\hline Frankreich & 222 & 39,869 & 43,760 \\
\hline Deutschland & 221 & 44,550 & 50,425 \\
\hline Slowenien & 215 & 23,654 & 34,408 \\
\hline Zypern & 209 & 24,976 & 37,023 \\
\hline $\begin{array}{l}\text { Vereinigtes König- } \\
\text { reich }\end{array}$ & 203 & 39,735 & 44,117 \\
\hline Belgien (Nord) & 198 & 43,582 & 46,553 \\
\hline Niederlande & 178 & 48,346 & 53,635 \\
\hline Lettland & 177 & 15,547 & 27,644 \\
\hline Irland & 175 & 70,638 & 75,539 \\
\hline Österreich & 175 & 47,290 & 49,869 \\
\hline Finnland & 168 & 46,017 & 44,333 \\
\hline $\begin{array}{l}\text { Schweiz (italie- } \\
\text { nisch) }\end{array}$ & 163 & 80,591 & 61,422 \\
\hline Dänemark & 156 & 56,444 & 49,883 \\
\hline Luxemburg & 148 & 105,803 & 106,374 \\
\hline Schweden & 140 & 53,218 & 51,475 \\
\hline $\begin{array}{l}\text { Schweiz (franzö- } \\
\text { sisch) }\end{array}$ & 139 & 80,591 & 61,422 \\
\hline Norwegen & 138 & 74,941 & 71,831 \\
\hline Schweiz (deutsch) & 121 & 80,591 & 61,422 \\
\hline
\end{tabular}

Aber wie herum man es auch betrachtet: Sorgen kann man sich in beiden Fällen machen ${ }^{8}$.

Denn es könnte ja sein, dass sich die Menschen in ärmeren Ländern eher vor den Fernseher zurückziehen, um sich von ihrer prekären Situation abzulenken. Der erhöhte TV-Konsum könnte also eine Folge der Armut sein. Es könnte jedoch auch sein, dass ein erhöhter TV-Konsum mehr Armut bedingt, z. B. weil weniger Arbeitszeit zur Verfügung steht oder weil mehr TV-Konsum nachweislich eine ungünstigere kognitive Entwicklung $[9,18,33]$ und eine geringere Bildung zur Folge hat [8]. Mehr TV bedeutet weniger Arbeit und weniger Bildung und beides bedeutet mehr Armut. Ich weiß nicht, worüber ich mir mehr Sorgen machen soll, die Verursachung von TV-Konsum durch Armut oder die Verursachung von Armut durch TV-Konsum - der Zusammenhang ist auf jeden Fall da und lässt sich nicht wegdiskutieren.

In vielfältigen Diskussionen über negative Auswirkungen von Bildschirmmedien habe ich über mehr als ein Jahrzehnt hinweg immer wieder erlebt, dass wie folgt argumentiert wurde: Diese Behauptung sei schon vor Jahrzehnten im Hinblick auf das Fernsehen erhoben worden und sei daher nicht ernst zu nehmen. Impliziert wird also (sonst funktioniert das Argument gar nicht), dass das Fernsehen keine negativen Auswirkungen habe. Dies traf nach vielfältigen Studien für Kinder und Jugendliche noch nie zu und ist nach den mittlerweile vorliegenden Daten auch für erwachsene Menschen nicht zutreffend. Es sei daher an dieser Stelle nochmals abschließend festgehalten: Das Fernsehen hat auch bei Menschen mit (weitgehend) abgeschlossener Gehirnentwicklung negative Auswirkungen auf die geistige Leistungsfähigkeit. Diese lassen sich nicht im Sinne eines reinen Verdrängungseffekts verstehen (Motto: Wer viel fernsieht, tut vieles andere, was gesund wäre, nicht). Vielmehr legen die vorliegenden Daten nahe, dass es sich beim Fernsehen um ein Verhalten handelt, das aufgrund seiner spezifischen Charakteristik (passive Aufnahme fragmentierter sensorischer Reize bei geringer Selbststeuerung bzw. geringem Top-Down-Informationsfluss und abwesender Handlungskonsequenz) der geistigen Leistungsfähigkeit eines Menschen schadet. Wie so oft macht auch hier die Dosis das Gift. Es ist eine Zeiterscheinung, dass wir heute in viel stärkerem Ausmaß zur Überdosierung neigen als noch vor wenigen Jahrzehnten, als es nur für ein paar Stunden täglich 2 oder 3 Programme zu sehen gab. Mit Hunderten von Programmen rund um die Uhr (die nicht zuletzt deswegen auch nicht anspruchsvoller wurden) sind wir einem Überangebot ausgesetzt, das die Überdosierung sicherlich begünstigt.

Ich kenne niemanden, der den Gedanken im Alter an Demenz zu erkranken, für attraktiv hält, etwa nach dem Motto: „Dann denke ich weniger und mache mir damit auch immer weniger Sorgen - auch was meinen eigenen Tod betrifft. Das ist doch viel an-

8 Keine Sorgen brauchte man sich zu machen, wenn die angeführte Korrelation gar nicht existierte, d. h. auf eine dritte Variable zurückgeführt werden könnte. So ist es beispielsweise bei der (über die Jahrzehnte glücklicherweise rückläufigen) Korrelation zwischen Einkommen und Schuhgröße, die sich auf die Variable „Geschlecht“ zurückführen lässt (Frauen verdienen weniger und haben kleinere Füße) und daher als Scheinkorrelation zu betrachten ist. Trotz längeren Nachdenkens und Diskutierens kann ich keine solche Variable erkennen. Prinzipiell ausschließen kann man sie nie. Sich aus diesem Grund keine Gedanken zu machen, wäre andererseits aus meiner Sicht jedoch ebenfalls unredlich. 
genehmer als diesen ganzen Prozess bewusst miterleben zu müssen. “Wenn aber so wirklich niemand denkt, dann müssen sich alle die Frage gefallen lassen, warum sie täglich einige Stunden daran arbeiten, dass es genau so kommt.

\section{Literatur}

[1] Aronson M. Does excessive television viewing contribute to the development of dementia? Med Hypotheses 1993; 41: 465-466

[2] Bakrania K, Edwardson CL, Khunti K et al. Associations Between Sedentary Behaviors and Cognitive Function: Cross-Sectional and Prospective Findings From the UK Biobank. Am J Epidemiol 2018; 187: 441-454

[3] Christakis DA, Ramirez JSB, Ferguson SM et al. How early media exposure may affect cognitive function: A review of results from observations in humans and experiments in mice. PNAS 2018; 115: 9851-9858

[4] Christakis DA, Ramirez JSB, Ramirez JM. Overstimulation of newborn mice leads to behavioral differences and deficits in cognitive performance. Sci Rep 2012; 2: 546

[5] Da Ronch C, Canuto A, Volkert J et al. Association of television viewing with mental health and mild cognitive impairment in the elderly in three European countries, Data from the MentDis_ICF65 + project. Ment Health Phys Act 2015; 8: 8-14

[6] Fancourt D, Steptoe A. Television viewing and cognitive decline in older age: findings from the English Longitudinal Study of Ageing. Scientific Reports 2019; 9: 2851

[7] Fogel J, Carlson MC. Soap operas and talk shows on television are associated with poorer cognition in older women. South Med J 2006; 99: $226-234$

[8] Hancox RJ, Milne BJ, Poulton R. Association of television viewing during childhood with poor educational achievement. Arch Pediatr Adolesc Med 2005; 159: 614-618

[9] Hinkley T, Verbestel V, Ahrens W et al.; für das IDEFICS Consortium. Early childhood electronic media use as a predictor of poorer wellbeing: a prospective cohort study. JAMA Pediatr 2014; 168: 485-492

[10] Hoang TD, Reiss ], Zhu N et al. Effect of Early Adult Patterns of Physical Activity and Television Viewing on Midlife Cognitive Function., Effect of early adult patterns of physical activity and television viewing on midlife cognitive function. JAMA Psychiatry 2016; 73: 73-79

[11] Holt-Lunstad J, Smith TB, Baker M et al. D Loneliness and social isolation as risk factors for mortality: A meta-analytic review. Perspectives on Psychological Science 2015; 10: 227-237

[12] Holt-Lunstad J, Smith TB, Layton JB. Social relationships and mortality risk: A meta-analytic review. PLoS Medicine 2010; 7: e1000316

[13] Lauricella AR, Cingel DP, Beaudoin-Ryan L et al. The Common Sense census: Plugged-in parents of tweens and teens. Common Sense Media 2017, San Francisco, CA. (http://cmhd.northwestern.edu/ wp-content/uploads/2017/04/common-sense-parent-census_whitepaper_new-for-web.pdf; abgerufen am 24.04.2019)
[14] Lillard AS, Drell MB, Richey EM et al. Further examination of the immediate impact of television on children's executive function. Dev Psychol 2015; 51: 792-805

[15] Lillard AS, Li H, Boguszewski K. Television and children's executive function. Adv Child Dev Behav 2015; 48: 219-248

[16] Lindstrom HA, Fritsch T, Petot $G$ et al. The relationships between television viewing in midlife and the development of Alzheimer's disease in a case control study. Brain Cogn 2005; 58: 157-165

[17] Livingston G, Sommerlad A, Orgeta V et al. Dementia prevention, intervention, and care. Lancet 2017; 390: 2673-2734

[18] Madigan S, Browne D, Racine N et al. Association Between Screen Time and Children's Performance on a Developmental Screening Test. JAMA Pediatr 2019; doi: 10.1001/jamapediatrics.2018.5056

[19] Rehbein F. Mediengewalt und Kognition: Eine experimentelle Untersuchung der Auswirkungen gewalthaltiger Bildschirmmedien auf Gedächtnis- und Konzentrationsleistung am Beispiel der Computerspielnutzung. Baden-Baden: Nomos Verlag, 2011

[20] Spitzer M. Arme, virtuelle Realität. Kleinkinder und elektronische Medien. Nervenheilkunde 2004; 23: 183-185

[21] Spitzer M. Vorsicht Bildschirm. Stuttgart: Klett; 2005

[22] Spitzer M. Fernsehen und Bildung. Nervenheilkunde 2005; 24 : 671-674

[23] Spitzer M. Achtung: Baby-TV. Nervenheilkunde 2007; 26: 1036-1040

[24] Spitzer M. Gemütlich dumpf. Nervenheilkunde 2009; 28: 334-346

[25] Spitzer M. Digitale Demenz. München: Droemer; 2012

[26] Spitzer M. Cyberkrank! München: Droemer; 2015

[27] Spitzer M. Schlaflos mit Blaulicht. Nervenheilkunde 2015; 34: 560-562

[28] Spitzer M. Einsamkeit. München: Droemer; 2018

[29] Spitzer M. Die Smartphone Epidemie. Stuttgart: Klett-Cotta; 2018

[30] Statista. 2019. Durchschnittliche tägliche Fernsehdauer in Deutschland in den Jahren 1997 bis 2018 (in Minuten) (https://de.statista. com/statistik/daten/studie/118/umfrage/fernsehkonsum-entwicklung-der-sehdauer-seit-1997/; abgerufen am 8.4.2019)

[31] Statista. 2019. Fernsehkonsum: Tägliche Sehdauer der Deutschen in Minuten nach Altersgruppen (03. April 2019) (https://de.statista. com/statistik/daten/studie/2913/umfrage/fernsehkonsum-der-deutschen-in-minuten-nach-altersgruppen/; abgerufen am 8.4.2019)

[32] Statista. 2019. Durchschnittliche tägliche Fernsehdauer in den europäischen Ländern in den Jahren 2016 und 2017 (in Minuten) (https:// de.statista.com/statistik/daten/studie/196083/umfrage/fernsehkonsum-sehdauer-in-ausgewaehlten-laendern-in-europa/; abgerufen am 8.4.2019)

[33] Walsh J], Barnes JD, Cameron JD et al. Associations between 24 hour movement behaviours and global cognition in US children: a cross-sectional observational study. Lancet Child Adolesc Health 2018; 2: 783-791

[34] Wang JY], Zhou DHD, Li J et al. Leisure activity and risk of cognitive impairment: The Chongqing aging study. Neurology 2006; 66: 911-913 